\title{
Evaluation of X-Ray Doses on Children, from Paediatric Hospitals in Sudan
}

\author{
K. E. M. Mohamadain ${ }^{1}$, S. M. Ibrahim ${ }^{1,2}$ \\ ${ }^{1}$ Department of Physics, Faculty of Science, Gassim University, Qassim, Saudi Arabia \\ ${ }^{2}$ Sudan University of Science and Technology, Khartoum, Sudan \\ Email: kouther@hotmail.com
}

Received May 26, 2013; revised June 26, 2013; accepted July 23, 2013

Copyright (C) 2013 K. E. M. Mohamadain, S. M. Ibrahim. This is an open access article distributed under the Creative Commons Attribution License, which permits unrestricted use, distribution, and reproduction in any medium, provided the original work is properly cited. In accordance of the Creative Commons Attribution License all Copyrights (C) 2013 are reserved for SCIRP and the owner of the intellectual property K. E. M. Mohamadain, S. M. Ibrahim. All Copyright (C 2013 are guarded by law and by SCIRP as a guardian.

\begin{abstract}
Children are more sensitive to radiation than adults, so radiation protection in paediatric radiology deserves special attention. This work estimates the effective doses and body organ doses due to chest examinations in infants and paediatrics. Two examination incidences, AP and PA for chest X-ray exposures were evaluated and compared with respect to the radiographic technique employed. This study was carried out in three paediatric hospitals in Sudan. The age intervals considered were $0-1$ year, 1 - 5 years, 5 - 10 years and $10-15$ years. The results obtained for organ doses and effective doses were calculated using a software package developed by the Radiological Protection Centre of the Saint George's Hospital, London. Effective dose values were also evaluated considering weight intervals from 1 - $10 \mathrm{~kg}$, 10 $20 \mathrm{~kg}, 20-30 \mathrm{~kg}$ and $30-40 \mathrm{~kg}$. Large discrepancies were encountered between the three hospitals, probably due to the different radiographic technique employed, calibration and maintenance of the X-ray equipment, technicians' expertise, and processing conditions.
\end{abstract}

Keywords: Effective Dose; Dose Cal Software; Body Organ Dose

\section{Introduction}

Quality Assurance Programmes in diagnostic radiology have been developed in several countries in the past 16 years, mainly in Europe [1,2]. However, the need for special QAP for paediatric patients was first realised early in the 1980s $[3,4]$. The main goals were to improve the diagnostic information and to reduce the patient dose to a minimum ALARA principle [5]. The efforts towards QA in paediatric radiology were at first dominated by the principle of justification and also by the concepts of "efficacy/efficiency" [6,7]. The WHO Report 757 compiled such principles for a number of common diseases in paediatrics and emphasised the term "rational use of diagnostic imaging" [8]. The second important principle of "optimisation" [9] is contemplated by the document "Quality Criteria for Diagnostic Radiographic Images in Paediatrics" [10] and an earlier developed document for adults, "Quality Criteria for Diagnostic Radiographic Images" [11].
One of the main aspects on the implementation of QA programs is the calibration and maintenance of X-ray equipment and well-trained technicians. QAP [12] also intends to reduce rejection rates, improve image quality, reduce doses imparted to patients and consequently, reduce costs of radiology departments.

Quality assurance in paediatric radiology is still more important, since it is known that children are more sensitive to radiation than adults [13]. Therefore, in this case, closer attention should be paid to improve the diagnostic information, reducing the child dose as much as possible [14] as well as choosing the most convenient type of projection.

\section{Materials and Methods}

This study was carried out in three paediatric hospitals in Sudan: Hospital A (university Hospital), Hospital B and Hospital C (general hospital). The age intervals considered were $0-1$ year, $1-5$ years, $5-10$ years and $10-15$ 
years and the weight intervals from $1-10 \mathrm{~kg}, 10-20 \mathrm{~kg}$, $20-30 \mathrm{~kg}$, and $30-40 \mathrm{~kg}$. In this work, two examination techniques were used, PA and AP projections.

The evaluation of the performance of the X-ray equipment in all three hospitals was performed by the Sudanese Atomic Energy Agency, however. Equipment maintenance is only corrective, not preventive.

The three hospitals are of medium size (around 140 beds each), however they have a very heavy workload, with approximately 8400 patients per month, each.

The examinations are performed by the technician and supervised by resident physicians. The main types of examinations are chest, skull and pelvis.

For each hospital and each projection, age, sex, weight and technical exposure parameters (tube voltage, current, time product, SSD) were recorded.

The X-ray machines used were FB-GT 22 (Hospital A), Siemens (Hospital B) and GE Beaver R201 (Hospital C). In this work, the number of patients was 31,54 and 44 for A, B and C hospitals, respectively. The technical parameters used according to the type of examination and the patient age, varied from 36 to $78 \mathrm{kV}$ for AP projection and from 36 to $70 \mathrm{kV}$ for PA projection. With respect to the transportable charge, the values ranged from 1.8 to $20 \mathrm{mAs}$ for AP projection and from 3 to 16 $\mathrm{mAs}$ for the PA projection.

A software package specially constructed for the evaluation of effective dose and body organ doses. Using this software, it possible to estimate patient doses know- ing the X-ray tube output that must be measured using a calibrated ionization chamber at $80 \mathrm{Kv}, \mathrm{FSD}$ equal to $100 \mathrm{~cm}$ and a transportable charge of $10 \mathrm{mAs}$. For the calculation of the Entrance Surface Dose, and the effective dose with the use of relation (1) and (2) below, it is necessary as well, the knowledge of the back scatter factor (BSF), field size and tube filtration.

$$
\begin{gathered}
E S D=\operatorname{Output}\left\{k V^{2} / 80^{2}\right\}\left\{100^{2} / F S D^{2}\right\} m A s / B S F \\
E D=E S D \times C f(E D)
\end{gathered}
$$

where:

The standard output factor in $\left(\frac{m G y}{m A s}\right)$ for the particular radiographic equipment used, measured under minimal scatter conditions at $1 \mathrm{~m}$ from the tube focus at nominal $80 \mathrm{kV}$; mAs is the product of tube current and exposure time. And $\mathrm{Cf}(\mathrm{ED})$ is NRPB factor used to convert the ESD to ED.

\section{Results and Discussion}

Results for effective doses according to weight are presented in Table 1, for the three hospitals.

The effective dose, which is directly related with the risk, depends on several factors: radiographic technique (kv, $\mathrm{mAs}$, etc.), processing conditions, equipment calibration and performance, film-screen sensibility and the technicians' expertise, among others.

Table 1. Values of effective doses according to weight for the three hospitals.

(a)

\begin{tabular}{cccccccc}
\hline Weight (kg) & kv & mAs & Eff. dose (msv) & sd (yEr+) & se (yEr+) & sum & N \\
\hline $0-10$ & $36-45$ & $1.8-3.0$ & 0.02163 & 0.00291 & $7.51 \mathrm{E}-04$ & 0.3244 & 15 \\
$10-20$ & $42-54$ & $3.0-3.6$ & 0.02285 & 0.00467 & 0.0019 & 0.1371 & 6 \\
$20-30$ & $45-57$ & $3.0-3.6$ & 0.02204 & 0.00439 & 0.00139 & 0.2204 & 10 \\
\hline
\end{tabular}

(b)

\begin{tabular}{cccccccc}
\hline Weight (kg) & kv & mAs & Eff. dose (msv) & sd (yEr+) & se (yEr+) & sum & N \\
\hline $0-10$ & 36 & $4.0-6.4$ & 0.37865 & 0.0608 & 0.01268 & 8.709 & 23 \\
$10-20$ & $36-38$ & $5.0-8.0$ & 0.31673 & 0.13595 & 0.04099 & 3.484 & 11 \\
$20-30$ & $38-40$ & $6.4-8.0$ & 0.26200 & 0.04101 & 0.01834 & 1.31 & 5 \\
$30-40$ & $38-44$ & $6.4-10$ & 0.33800 & 0.07622 & 0.03409 & 1.69 & 5 \\
\hline
\end{tabular}

(c)

\begin{tabular}{cccccccc}
\hline Weight (kg) & kv & MAs & Dose (msv) & sd (yEr+) & se (yEr+) & sum & N \\
\hline $0-10$ & $53-60$ & $6.0-10.0$ & 0.15666 & 0.05101 & 0.01237 & 2.6632 & 17 \\
$10-20$ & $50-70$ & $6.0-12.0$ & 0.17705 & 0.12875 & 0.02954 & 3.364 & 19 \\
$20-30$ & $63-78$ & $10.0-16.0$ & 0.24638 & 0.24444 & 0.08642 & 1.971 & 8 \\
\hline
\end{tabular}


From the results presented in Table 1, where we take into consideration only the children's weight, it can be seen that large differences in doses are present from one hospital to the other. The greatest difference registered was for the weight interval from $0-10 \mathrm{~kg}$. In hospital B, the mean effective dose was $0.379 \mathrm{msv}$ while in hospital A it was only $0.022 \mathrm{msv}$. Therefore, a difference of $1700 \%$ in the effective dose was detected between the two hospitals. It means that, children of up to $10 \mathrm{~kg}$ that take a chest X-ray in hospital B are likely to receive 17 times more radiation than those who take the same radiograph in hospital A.

Still analysing the results from Table 1, it can be seen that the radiographic technique for the three hospitals differs both, in kv and mAs. For the weight interval from $0-10 \mathrm{~kg}$, where the largest difference in effective dose was detected, A and B hospitals use the approximately the same $\mathrm{kV}$ range. However, the mAs, ranges from 1.8 3.0 (mean value of 2.4) in hospital A, while in hospital B it ranges from 4.0 to 6.4 (mean value of 5.2). The mean $\mathrm{mAs}$ value in hospital B is at least doubled as compared to hospital A. This could be one of the causes of the higher effective doses.

On the other hand, analysing the radiographic technique of hospital $\mathrm{C}$, for the same weight interval, it is seen that the $\mathrm{kV}$ ranges from $6-10$ (mean value of 8 ), being consequently even higher than hospitalB. However, the effective dose for this Hospital is not the highest. In this case, the relatively low effective dose could be accounted for better equipment (more modern and/or with appropriate maintenance), more adequate processing conditions, and/or technicians better trained.

Table 2 presents the results of effective dose in terms of age intervals.

Analysing effective doses for the three hospitals, it is found that the worst situation is also for the smallest babies, from 0 - 1 year, and Large discrepancies were encountered between A and B hospitals.

In Table 3, organ doses are shown for the three hospitals, for AP and PA projections.

Concerning organ doses, for AP projection presented in Table 3; once more the highest values were encountered in hospital B. Comparing B and hospitals A, the organs that presented the highest doses, in the two hospitals were: breast $(0.272$ and $0.015 \mathrm{mSv})$, thymus $(0.224$ and 0.013$)$, heart $(0.150$ and 0.008$)$, thyroid $(0.130$ and $0.008)$ and lungs $(0.121$ and 0.007$)$ respectively. For these organs the increase in doses from hospital A to hospital B is again around 17 to 18 times.

Analysing the results from the three hospitals and now considering PA projection, as compared to AP projection, much higher doses for the PA projection were

Table 2. Effective doses according to age interval for the three hospitals.

(a)

\begin{tabular}{ccccccc}
\hline Age in Year & Eff. dose (msv) & sd $(\mathbf{y E r}+)$ & se (yEr+) & sum & N & Projection \\
\hline $0-1$ & 0.01963 & 0.00307 & 0.00125 & 0.1178 & 6 & AP \\
$1-5$ & 0.02280 & 0.00324 & 0.00097 & 0.2510 & 11 & AP \\
$5-10$ & 0.02381 & 0.00377 & 0.00142 & 0.1667 & 7 & PA \\
$10-15$ & 0.02091 & 0.00405 & 0.00153 & 0.1464 & 7 & PA \\
\hline
\end{tabular}

(b)

\begin{tabular}{ccccccc}
\hline Age in Year & Eff. dose (msv) & sd (yEr) & se (yEr+) & sum & N & projection \\
\hline $0-1$ & 0.33017 & 0.06630 & 0.02707 & 1.981 & 6 & AP \\
$1-5$ & 038445 & 0.08815 & 0.01879 & 8.458 & 22 & AP \\
$5-10$ & 0.27900 & 0.09290 & 0.03097 & 2.511 & 9 & PA \\
$10-15$ & 0.32043 & 0.07351 & 0.02779 & 2.243 & 7 & PA \\
\hline
\end{tabular}

(c)

\begin{tabular}{ccccccc}
\hline Age in Year & Eff. dose (msv) & sd (yEr+) & se (yEr+) & sum & N & projection \\
\hline $0-1$ & 0.13952 & 0.04375 & 0.01383 & 1.3952 & 10 & AP \\
$1-5$ & 0.20900 & 0.14174 & 0.04092 & 2.5080 & 12 & AP \\
$5-10$ & 0.23258 & 0.24137 & 0.08046 & 2.0932 & 9 & PA \\
$10-15$ & 0.15871 & 0.04621 & 0.01747 & 1.1110 & 7 & PA \\
\hline
\end{tabular}


Table 3. Organ doses calculated with the use of a dosimetry software for the three hospitals for both, AP and PA projections. Body organ dose in (mSv) for (AP projection)and (PA projection).

\begin{tabular}{cccccccc}
\hline \multirow{2}{*}{ projection } & \multicolumn{2}{c}{ Hospital A } & \multicolumn{2}{c}{ Hospital B } & \multicolumn{3}{c}{ Hospital C } \\
\cline { 2 - 7 } & AP & PA & AP & PA & AP & PA \\
\hline Breasts & 0.01511 & 0.00418 & 0.27172 & 0.01177 & 0.12857 & 0.00955 \\
Thymus & 0.01262 & 0.00291 & 0.22368 & 0.00522 & 0.05545 & 0.00251 \\
Heart & 0.00855 & 0.00263 & 0.14972 & 0.0152 & 0.00396 & 0.02324 \\
Thyroid & 0.00802 & 0.00291 & 0.12946 & 0.00204 & 0.0001 & 0.00004 \\
Lungs & 0.00696 & 0.0056 & 0.12123 & 0.06619 & 0.0003 & 0.00002 \\
\hline
\end{tabular}

found in some body organs due to their location with respect to the direction of the incident beam. This fact is of special concern in paediatric radiology because little babies are more likely to suffer the effects of radiation and have longer life expectation. Therefore, physicians, when deciding for a certain type of projection, they should be aware of these differences to be able to decide which the most adequate technique to be chosen is. Probably, in some situations, not only scattered radiation is measured, but also the direct beam, since new-borns bodies are very small and are almost completely irradiated during examination. In this particular case, proper collimation would certainly reduce doses significantly.

\section{Conclusions}

In hospital $\mathrm{B}$, the doses are always higher compared to hospital A, so care should be taken in this hospital to avoid the potential health hazards to paediatrics patients due to exposure during X-ray diagnostic. On the other hand, hospital A presents the lowest dose values for all age range. The reason is that Hospital A is paediatrics university hospital with a very well trained staff to work with neonates and children in general, the other two hospitals considered in this work are general hospital not dedicated for paediatrics, their staff have no special training to work with children, their equipments and procedure are normally adapted for adults.

In Table 3, the BOD can be seen for Ap and PA projection respectively, and it has been seen that the AP projection delivers high dose to the Brest and thyroid and should be avoided whenever possible specially for girls.

By the results shown above, we can conclude that a lot can be done to reduce doses in radiology, being of special concern of paediatric departments. The importance of the implementation of Quality Assurance Programs in radiology departments should be stressed because most of the non-conformal working conditions that are here reflected by the differences in doses could be controlled if a QAP was implemented on a regular basis. X-ray equipment properly calibrated, with a good maintenance schedule, correct processing conditions, adequate screenfilm combination and well-trained technicians are among the most important items to be considered. It is also of great importance to instruct physicians about the basic principals of radioprotection so that they would be aware of the correct methods to reduce radiation risks in infants.

\section{REFERENCES}

[1] DIN 6868/1, "Sicherung der Bildqualität in Röntgendiagnostischen Betrieben; Allgemeines," Beuth Verlag GmbH, Berlin, 1985.

[2] H. S. Stender and F. E. Stieve, "Praxis der Qualitätskontrolle in der Röntgendiagnostik," Gustav Fischer Verlag, Stuttgart, New York, 1986.

[3] ICRP, "Recommendations of the International Commission on Radiological Protection," Publication 26, Pergamon Press, Oxford, 1977.

[4] H. Fendel, "Symposium: The Status of Paediatric Radiology in Europe. The Principles for Rational Use and Optimisation of Diagnostic Imaging in Paediatrics," 27th Congress of ESPR, Munich, 1990.

[5] H. Fendel, K. Schneider, C. Bakowski, J. Glas, K. Drews and M. M. Kohn, "The Efficacy of Diagnostic Radiation in Paediatrics," 2nd Report, Bundesministerium für Umwelt, Naturschutz und Reaktorsicherheit, Bonn, 1986.

[6] World Health Organisation-Study Group, "Rational Use of Diagnostic Imaging in Paediatrics," WHO Technical Report Series 757, WHO, Geneva, 1987.

[7] H. Fendel, K. Schneider, C. Bakowski and M. M. Kohn, "Specific Principles for Optimisation of Image Quality and Patient Exposure in Paediatric Diagnostic Imaging. Optimisation of Image Quality and Patient Exposure in Diagnostic Radiology," In: B. M. Moores, et al., Eds., BIR 20, British Institute of Radiology, London, 1989, pp. 91-110.

[8] CEC, "Quality Criteria for Diagnostic Radiographic Images in Paediatrics," Working Document No. XII/307/91, 1992.

[9] NRPB, "Patient Dose Reduction in Diagnostic Radiology,” NRPB 1, No. 3, HM50, London, 1990 
[10] CEC, "Quality Criteria for Diagnostic Radiographic Images," 2nd Edition, Working Document No. XII/173/90, 1990.

[11] A. C. P. Azevedo, A. Yacovenco, O. D. Goncalves, H. A. Koch and E. T. Tonomura, "Avaliacao do Funcionamento do Servico de Radiodiagnostico do HUCFF-UFRJ," Radiologia Brasileira, Vol. 32, 1999, pp. 309-312.

[12] A. C. P. Azevedo, O. D. Goncalves, S. R. Oliveira, M. O. Silva and L. A. G. Magalhaes, "Quality Control of Viewing Boxes,” Physica Medica XVII/2, 2001, pp. 89-92.
[13] H. Fendel, K. Schneider, H. Schöfer, C. Bakowski and M. M. Kohn, "Optimisation in Pediatric Radiology: Are There Specific Problems for Quality Assurance in Pediatric Radiology?" In: B. M. Moores, et al., Eds., Technical and Physical Parameters for Quality Assurance in Medical Diagnostic Radiology: Tolerances, Limiting Values and Appropriate Measuring Methods, BIR 18, British Institute of Radiology, London, 1985, pp. 159-165.

[14] K. Schneiden, "Evaluation of Quality Assurance in Pediatric Radiology," Rad Prot Dos 57, 1995, pp. 119-123. 